\title{
Erratum to: A metallic buildup cap for glass rod dosimeters in radiotherapeutic in vivo dosimetry
}

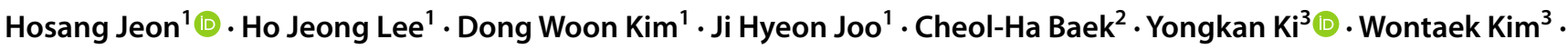 \\ Dahl Park ${ }^{4}$ Jiho $\mathrm{Nam}^{4} \cdot$ Dong Hyeon Kim ${ }^{4}$
}

Published online: 7 February 2022

(c) The Korean Physical Society 2022

\section{Erratum to: \\ Journal of the Korean Physical Society (2021) 78:837-841 \\ https://doi.org/10.1007/s40042-021-00105-2}

This paper included an unrelated grant number in Acknowledgments on page 840 because of the corresponding author's mistake.

The correct Acknowledgements should be read:

This research was supported by the Basic Science Research Program through the National Research Foundation of Korea (NRF) funded by the Korean government (2020R1C1C1013300) and a 2021 research grant from Pusan National University Yangsan Hospital.

The original article can be found online at https://doi.org/10.1007/ s40042-021-00105-2.

Yongkan Ki

apex7171@hanmail.net

1 Department of Radiation Oncology and Research Institute for Convergence of Biomedical Science and Technology, Pusan National University Yangsan Hospital, Yangsan, South Korea

2 Department of Radiological Science, Kangwon National University, Samcheok, South Korea

3 Department of Radiation Oncology, Pusan National University School of Medicine, Yangsan, South Korea

4 Department of Radiation Oncology, Pusan National University Hospital, Busan, South Korea
Publisher's Note Springer Nature remains neutral with regard to jurisdictional claims in published maps and institutional affiliations. 